Recibido: 04/05/2020 --- Aceptado: 03/08/2020 --- Publicado: 15/11/2020

\title{
COMUNICACIÓN SOSTENIBLE Y SOCIEDAD 2.0: PARTICULARIDADES EN UNA RELACIÓN DE TRES DÉCADAS
}

\section{SUSTAINABLE COMMUNICATION AND 2.0 SOCIETY: ODDITIES DERIVED FROM A THREE-DECADE RELATIONSHIP}

Eva Aladro Vico': Universidad Complutense de Madrid. España. ealadro@ucm.es

\section{RESUMEN}

El propósito de este artículo es ahondar en la materia de la comunicación sostenible, sus implicaciones y sus relaciones con otras materias dentro de las sinergias creadas por las nuevas tecnologías y por la creciente importancia de la sostenibilidad como valor dentro de la sociedad moderna. La comunicación sostenible se puede entender, a burdos brochazos, como comunicación corporativa o lobbying/cabildeo aplicado a la causa de la sostenibilidad. Sin embargo, es este último detalle el que le aporta características que la distinguen críticamente de otras ramas de la comunicación, y que interactúa de forma extraordinaria con las nuevas realidades TIC y 2.0.

PALABRAS CLAVE: TIC - Comunicación sostenible - 2.0 - Sostenibilidad Infoxicación

\section{ABSTRACT}

The purpose of this article is to delve into the subject of sustainable communication, its implications and its relationships with other subjects within the synergies created by new technologies and by the growing importance of sustainability as a value within modern society. Sustainable communication can be understood, in rough strokes, as corporate communication or lobbying applied to the cause of sustainability. However, it is this last detail that provides the characteristics that critically distinguish it from other branches of communication, and the interaction in an extraordinary way with the new ICT and 2.0 realities.

KEY WORDS: CIT - Sustainable communication - 2.0 - Sustainability - Infoxication

1 Eva Aladro Vico: Profesora titular del Departamento de Periodismo y Nuevos Medios, Facultad de Ciencias de la Información, Universidad Complutense de Madrid. Autora de los libros Las Diez Leyes de la Teoría de la Información, Teoría de la Información y la Comunicación Efectiva http://orcid.org/0000-0003-1986-8312. https://www.researchgate.net/profile/Aladro_Eva 


\section{COMUNICAÇÃO SUSTENTÁVEL E SOCIEDADE 2.0: PARTICULARIDADES EM UMA RELAÇÃO DE TRÊS DÉCADAS}

\section{RESUMO}

O propósito deste artigo é mergulhar na matéria da comunicação sustentável, suas implicações e suas relações com outras matérias dentro das sinergias criadas pelas novas tecnologias e pela crescente importância da sustentabilidade como um valor dentro da sociedade moderna. A comunicação sustentável pode se entender, a grosso modo, como comunicação corporativa ou lobbying aplicado à causa da sustentabilidade. Porém, é este último detalhe que aporta as características que aS distinguem criticamente de outras ramificações da comunicação, e que interagem de forma extraordinária com as novas realidades TIC y 2.0.

PALABVAS CHAVE: TIC - Comunicação sustentável - 2.0 - Sustentabilidade Infoxicação

\section{Cómo citar el artículo:}

Aladro Vico, E. (2020). Comunicación sostenible y sociedad 2.0: particularidades en una relación de tres décadas. [Sustainable communication and 2.0 Society: oddities derived from a three-decade relationship]. Revista de Comunicación de la SEECI, 53, 37-51. doi: https://doi.org/10.15198/seeci.2020.53.37-51

Recuperado de http://www.seeci.net/revista/index.php/seeci/article/view/642

\section{INTRODUCCIÓN}

Entendemos por "comunicación sostenible" aquella cuyo objeto de existencia es el fomento del desarrollo humano y social sostenible, asociada a los valores de, por supuesto, sostenibilidad, diálogo, transparencia, gobernabilidad y transparencia por su vocación de hacer sostenible el sistema entero. El concepto está en concordancia con otros similares que aparecen en la sociedad del post-decrecimiento, y encaja en la filosofía de los autores y analistas que piden que la sostenibilidad sea el criterio vital en todos los aspectos de actividad humana, crucialmente, por ello, también en la comunicación.

Dentro de esta idea, el agente moderador de la sociedad lo constituyen los medios, en su papel de investigadores y denunciantes de la actividad pública ilegítima, así como de educadores e informadores potenciales en materia de sostenibilidad. La comunicación es un vórtice de un triángulo de sostenibilidad que comprende también la economía y la estructura social.

Los medios constituyen la lupa a través de la cual la sociedad ve a las organizaciones, siendo esta perspectiva crucial a la hora de determinar si les es renovada la confianza del público en sus mensajes y declaraciones. No es importante tan sólo que la actividad de la empresa, ONG o institución sea correcta: además debe parecerlo. Y podría perfectamente solo parecerlo. No sirve de nada socialmente- obrar de forma recta si dicha rectitud se ignora. Los medios recuperan 
así la función de vigilancia del entorno que en su día tuvieron en su modelo teórico clásico.

\section{OBJETIVOS}

El objetivo de este artículo es establecer un estado de la cuestión actualizado de la comunicación sostenible con respecto a las implicaciones que puedan aplicársele en virtud de la implantación de nueva tecnología y de los fenómenos que lleva aparejados: la posverdad y toda su cohorte de fenómenos acólitos, en relación a su comparación con la comunicación y la responsabilidad social corporativas. Pretendemos establecer claramente la diferenciación entre estos fenómenos así como sus múltiples puntos en común, cómo todos ellos se interrelacionan, para llegar a conclusiones claras sobre la nomenclaturización, objetivos y características de este tipo de comunicación bajo dichas premisas, como medio de determinar el camino futuro a seguir de esta rama de la comunicación.

\section{METODOLOGÍA}

Nos proponemos llevar a cabo una revisión suficiente de fuentes y literatura de un amplio espectro temático que nos permita abarcar con autores de calidad este tema tan, por otra parte, insuficientemente tratado en relación a su vertiente comunicativa. Compararemos las fuentes, descartando las que no hagan afirmaciones relacionadas o lógicas e entretejiendo la información para alcanzar sinergias significativas que den lugar a una comprensión simplificada pero completa de la materia y sus potencialidades, sin menoscabo del rigor académico debido. Se valorarán las aportaciones de cada fuente y se imbricarán en un texto comprensible y estructurado que permita que un flujo natural de conclusiones sea alcanzado de forma orgánica.

\section{DESARROLLO}

Para que un hecho sea reconocido por el público, es preciso que algo esté presente en los medios (o en las Redes, como nos recuerda Caldevilla (2014, p. 1286):

Ya hemos hablado de que las Redes Sociales son un canal más, pero que facilitan la participación ciudadana en los asuntos públicos, permitiendo una democracia más directa y sana aunque éste no fuera su objetivo original, pues "...las Redes Sociales nacen como una reunión de personas, conocidas o desconocidas, que interactuarán entre sí, redefiniendo al grupo y retroalimentándolo "pero dada su capacidad de influir también convendremos que "...gracias a Internet y las Redes Sociales se puede modificar una conducta, crear nuevos movimientos de opinión, partidos políticos, plataformas reivindicativas, promover manifestaciones, crear grupos de apoyo a causas concretas o conseguir crear una moda que genere el consumo de un determinado producto. La ley está cercenando cada vez más este tráfico de información cuando su fin atenta contra los intereses del partido en el Gobierno. La relación entre usuarios de plataformas como las Redes Sociales 
pasa de ser vertical a horizontal, posibilitando una igualdad figurada, propiciatoria de que cualquier internauta se convierta en emisor, produciendo sus propios contenidos, y transmisor/receptor de información.

Si algo no forma parte de lo nuclear en los medios y las Redes sociales (que no han hecho sino ampliar el espectro previo de lo nuclear) en la práctica deja de existir. A ello se agrega la percepción de verdad que se tiene ante algo publicado -ya sea en los medios o en las redes-. El problema actualmente se ha extendido, como apunta también Caldevilla (2013, p. 40) en sus trabajos sobre la infoxicación:

Por tanto, la "infoxicación", o la intoxicación de la información por sobreabundancia, es una fórmula de la intoxicación intelectual producida por un exceso de información, que, paradójicamente, no ayuda sino distorsiona.

El exceso de información que se produce merced al alud de datos disponible hoy en día, genera una dificultad en el receptor (que se convierte en receptor a su vez) para asimilar tanto volumen en tan poco tiempo, sin contar con el debido reposo para valorar los contenidos ni su grado de veracidad, aunque estos estén dotados, generalmente, de una aparente alta verosimilitud. Una consecuencia de ello es la conversión del tiempo en un bien muy escaso y la búsqueda de la atención de la audiencia como una de las prioridades en Internet. De hecho, hoy existen empresas especializadas en posicionar las web de sus clientes en los primeros puestos de buscadores tipo Google, Bing o Baidu.

La "sobreinformación", no obstante, no es algo novedoso. Diderot afirmaba en su Enciclopedia en 1772 que, mientras los siglos continuaran desarrollándose, podría predecir que llegaría un momento en que sería casi imposible aprender cualquier cosa ya que cualquier fragmento de la verdad quedaría escondido en una inmensidad de volúmenes encuadernados

Los autores internacionales señalan igualmente este gravísimo problema que está marcando el desarrollo de la sociedad digital 2.0: como indica Sinan Aral y sus colegas investigadores ( Soroush, 2018), todo el sistema de comunicación digital de las redes trabaja para generar el engaño más perfecto -así en los nuevos Deep fakes y en las Fakes news-, de manera que la comunicación tóxica, diseñada para la manipulación emocional y para lo que Lanier (2018) denomina el condicionamiento cognitivo de los individuos, conspiran y se convierten en el eje del crecimiento insostenible de la esfera comunicativa, en ese conjunto de elementos que favorece un desarrollo insostenible del sistema social.

Sin embargo, una consecuencia que también se puede extraer es que vivimos la época de la democracia persuasiva no vinculada a los conceptos de participación democrática levantados por Córdova Jaimes y Ávila Hernández (2017); es decir vivimos en un tiempo en el que la persuasión y la manipulación se convierten en las principales herramientas de acción de los agentes políticos y de la comunicación sobre la escena pública, resaltando con ello el papel secundario otorgado a la honestidad intelectual y a la veracidad. Como advierten Niño et ál. (2017, p. 91): 
Estamos, pues, ante un escenario preocupante en el mejor de los casos: aquel en el que el público es manipulado a través del propio público, y de medios estrictamente anónimos: los usuarios. En sus manos está, cada vez más, la selección final de información, pero todo parece indicar que seguimos lejos del momento cultural en el que el público sea capaz de ejercer esta función de forma crítica.

Por eso, en este contexto, el periodismo y los nuevos y viejos medios de comunicación ven también reforzado su papel difusor, educacional y sensibilizador en defensa del público, en relación a la infoxicación creciente y a la forma en que amenaza con enterrar los problemas sociales auténticos. Como acota Hernández Rubio (2019, p. 38):

Es necesario inculcar una ética posmoderna en cada momento de comunicación virtual entre dos o más personas. En el caso de la profesión informativa, hay que huir de esos homo ludens y homo oeconomicus para promover el hombre moral, capaz de ofrecer a su semejante unos contenidos verídicos alejados del espectáculo mediático.

El concepto mismo de desarrollo sostenible data de 1987, y aparece en un informe de Naciones Unidas, que establece el derecho a un ambiente sano y apto para el desarrollo para que la producción satisfaga las necesidades de las generaciones presentes sin comprometer las de las futuras. Animaba a las autoridades a contemplar dicho derecho junto con el derecho a la información y la educación ambiental, apelando a los medios -pre-2.0- a ser rigurosos en sus informaciones. Una apelación si cabe más importante -precisamente ante el fenómeno de la infoxicación y los bulos, como nos recuerda Mazo (2016): el rumor actúa en muchos de los casos como un mecanismo de defensa verbal para los individuos de un determinado grupo. [...] En el entorno de los medios de comunicación, los deseos del mal ajeno serán un contexto propicio para la creación y difusión de este tipo de mensajes. Sobre los riesgos de comunicación asociados a las redes sociales, Bustos-Martínez (2019, p. 28) añade:

La extensión de la red de redes ha provocado que su alcance e influencia resulte prácticamente ilimitada. Las esperanzas residen en que internet, y más en concreto las redes sociales, se configuren como potentes herramientas que colaboren en la creación de una sociedad más participativa en materia política y social. La libertad, bien entendida, es el concepto clave que guía el mundo digital y que ofrece la posibilidad de acceder a unas fuentes de información diferentes a las tradicionales y, teóricamente, más alejadas del control de lo político, económico y financiero.

La capacidad de la especie humana para manipular el entorno y ajustarlo a sus necesidades no tiene precedentes. $Y$ el aumento demográfico no ha hecho sino incrementar dicho impacto, como bien suscriben Barrientos et ál. (2017) de la mano con el desarrollo tecnológico, el consumismo y la interconectividad a una escala nunca vista. Todo ello nos lleva a la conclusión de que nuestro impacto en la tierra es, cada vez más, global. Y nos impele a buscar soluciones globales integradas al actuar sobre la huella ecológica. Servaes $(2012$, p. 18) resume: 
Mientras en el pasado éramos capaces de aumentar la producción de alimentos después de unos pocos ciclos de cultivo, o de establecer ingresos creando empresas en un par de años, los 'nuevos' problemas a los que nos enfrentamos pueden llevar años, y en el caso del cambio climático, muchas generaciones, para que la comunidad mundial pueda resolverlos. ¿Cómo podemos construir consenso y lograr el intento altruista de la generación actual de consumir menos, rebajar el conflicto, y someternos nosotros mismos a investigación médica para que las futuras generaciones que nos sobrevivan puedan heredar un planeta habitable?

Los métodos ya testados y probados de la agricultura extensiva, la movilización social, la participación comunitaria y la negociación multilateral difícilmente tendrán éxito por sí mismos si estos problemas sistémicos crecen con severidad y las personas caen en el innato instinto humano de la autopreservación, compitiendo aún más ferozmente por los reducidos recursos naturales, con violencia radical, y se resistirán a los principios hipocráticos de compartir las limitadas reservas de vacunas y medicamentos, acumularán energía y agua, y cerrarán los mercados al comercio internacional.

No tenemos estrategias adecuadas para empezar a revertir estos 'nuevos' y muy complejos desafíos. Por tanto, después de haber resumido brevemente el pasado de este sector, y subrayado algunos asuntos clave para el futuro, concluiremos con una lista de desafíos tanto para académicos como para profesionales de la Comunicación para el Cambio Social Sostenible (CSSC).

Acotar la extensión y forma de las políticas de desarrollo sostenible pasa necesariamente por medir y dar forma a estas de cara a hacerlas encajar mejor en las distintas sensibilidades de cada una de las partes que integran un sistema complejo y global, dando herramientas al poder público y a la iniciativa privada en el sentido de las políticas de desarrollo sostenible. El concepto mismo ha cambiado y se ha ampliado desde 1987, adhiriendo dimensiones económicas, ambientales, sociales y relativas a las instituciones. Sus necesidades comunicacionales también han cambiado, ante el auge de la comunicación emotiva como medio de persuasión social, tal como apuntan Mendieta y Estrada (2017, p. 154):

La comunicación política que se establece a partir de Internet en las redes sociales y otros recursos tecnológicos, constituye un cambio en la manera de interactuar; hacia la informalidad. Esta nueva tendencia ha construido el fenómeno del politaiment e infotaiment como recursos emotivos apoyados en la tecnología digital

En este desarrollo, los medios (tanto tradicionales como 2.0) son el factor clave de difusión, educación, intercambio y sensibilización para los distintos agentes sociales responsables de gestionar esta transición al desarrollo sostenible. Recordando al ya citado Hernández-Rubio, apuntamos coincidiendo con Soengas et ál. (2019, ap. 6.5) que: 
El 74\% de los estudiantes que consultan varios medios o varias Redes sociales sólo consumen información para saber qué sucede en su entorno y en el mundo, pero no cuestionan el tratamiento informativo de las noticias. No tienen una actitud crítica frente al enfoque de los hechos, ni muestran interés por conocer puntos de vista alternativos.

Como hemos estudiado en otra parte, la alfabetización crítica de los jóvenes tiene en el campo de la comunicación digital un enorme terreno de exploración, que abarca nuevos lenguajes, nuevos modos de concebir los espacios y los soportes de la comunicación, nuevas formas cooperativas de comunicación y el uso creador de la comunicación política (Aladro et al, 2018).

Efendy Maldonado (2019, p. 22) nos recuerda:

La particularidad del campo de la comunicación requiere, en su constitución transdisciplinar, de una apertura rigurosa, que sepa articular a partir de lo múltiple/teórico su complejidad argumentativa, y crezca en la diversidad de su particularidad, a partir de traducciones y montajes consistentes, relevantes y transcendentes en el conjunto existencial de las ciencias. Los sistemas transmedia y multimedia contemporáneos son centrales para las problematizaciones teóricas en el área; al mismo tiempo, son insuficientes para dar cuenta de la complejidad.

Para hablar propiamente de campos de comunicación debemos recordar, aunque sea a nivel epidérmico, que los nuevos vehículos de consumo de información modificarán la forma de acceso a la misma (Caldevilla, 2010, p. 35):

El último punto por desarrollar y que depende plenamente de los avances tecnológicos que los nuevos tiempos nos ofrecerán, se ubica en las mayores posibilidades de expresión que vehicularán los nuevos terminales en telefonía y la teoría de la pantalla unificada (televisión, radio, móvil, Internet...).

Todo lo anterior sólo podrá cobrar vida, no sólo si forma parte de lo nuclear (tendencias, modas...) en los Medios (Redes sociales incluidas) sino que esté presente en la agenda política ( $y$ después en la de eventos) de manera que los programas electorales (aunque mucho más citados que cumplidos) los contemplen como una promesa de actuación futura en los distintos gobiernos, incluyendo el efecto imitación o dominó por el que si un partido acciones de sostenibilidad 'obligue' a los demás a tomar postura.

Los medios de comunicación y Redes sociales pueden contribuir a mostrar la cara más amable de los candidatos que nos representan y que deben ser ejemplo de formación en valores, convicciones políticas y sociales. Por otro lado, los debates pueden ser una plataforma ideal para aportar soluciones a los intereses de la ciudadanía.

Nos planteamos como posible campo de investigación el modelo paralelo que parece que cada vez tiene más aceptación y participación por parte del electorado: las Redes sociales y el seguimiento en los debates políticos televisados. La bidireccionalidad puede ser un factor relevante que alienta el 
debate entre los ciudadanos y los partidos políticos (Barrientos et ál, 2019, p. 26).

\subsection{Clasificando la comunicación sostenible}

Es necesario distinguir todas ellas de las prácticas de Greenwashing (Miller, 2017) que han generado un estilo cultural de gran difusión pero de efectividad nula en términos de objetivos de sostenibilidad. El blanqueo de prácticas económicas y productivas contaminantes se sitúa justamente en el extremo opuesto de la comunicación sostenible, y es uno de sus principales peligros, porque puede convertir este objetivo en una simple estrategia de mercado que genere el efecto inverso, sustentando prácticas contaminantes o exterminadoras en el mundo de la producción y el consumo.

Para ello, vamos a discriminar diversos tipos de comunicación sostenible, que puedan resultar útiles para entender mejor el concepto.

Sobre la base de la comunicación corporativa y de la responsabilidad social corporativa, la comunicación sostenible se divide en tres tipos de enfoques u objetivos generales importantes a tener en cuenta:

- Comunicación y sostenibilidad

- Comunicación SOBRE sostenibilidad

- Comunicar sostenibilidad

- Comunicar PARA la sostenibilidad

\subsubsection{Comunicación y sostenibilidad}

La comunicación es de capital importancia para las estrategias de sostenibilidad. Allí donde existe una falta de comunicación interna, será difícil aplicar cambios en términos de prácticas sostenibles.

Adicionalmente, la comunicación externa (con los clientes, los socios y la comunidad) es una necesidad de las estrategias de comunicación sostenible. De lo contrario, se expone a pérdidas en términos de ventas y beneficios. La razón de todo esto puede explicarse considerando varios puntos: primero, la importancia del discurso social a la hora de proveer de legitimidad al desarrollo sostenible (Newig et ál., 2013). Segundo, la naturaleza altamente compleja de la materia de la sostenibilidad, que requiere de un abordaje específico (Newig et ál., 2008).

Típicamente, los asuntos relativos a la sostenibilidad son reconocibles por su complejidad e incertidumbre, aumentando la importancia de la comunicación a la hora de entregar información entre los diversos agentes del proceso productivo. Debido a la importancia de las decisiones al respecto, Funtowicz y Ravetz (1993) sugirieron nuevas aproximaciones científicas, incluyendo alta implicación en comunicación y diálogo, implicación de los accionistas en la extensión informativa, así como énfasis en los valores sociales. Adicionalmente, los objetivos de la 
sostenibilidad aparentan ser ambivalentes en términos de implicación en conflictos de intereses o valores. Llegados a este punto, la comunicación se vuelve esencial para crear una comprensión general de los valores sociales de la sostenibilidad, determinando algunos objetivos concretos que deben ser seguidos (Newig, 2013).

Por último, pero no menos importante, la implementación de medidas para el desarrollo sostenible es característicamente difícil, puesto que las capacidades para administrarlo se encuentran dispersas en multitud de actores sociales y múltiples niveles de decisión. Así pues, la coordinación basada en estructuras de red sería útil a la hora de permitir un debate y negociación eficaces, así como aprendizaje social, que permita contribuir a la implementación de estrategias de desarrollo sostenible. (Newig et ál., 2013).

\subsubsection{Comunicación sobre Sostenibilidad}

Abarca todos los procesos donde asuntos relativos a informaciones, interpretaciones y valoraciones relativas a la sostenibilidad son discutidas y expuestas. Asuntos y cuestiones que son transformadas y compartimentadas en un entorno de comunicación horizontal a múltiples niveles: desde el interpersonal del "cara a cara", hasta la comunicación de masas a través de los Media (Neidhardt, 1993).

La comunicación sobre sostenibilidad cubre las cuestiones de percepción de la sostenibilidad en tanto que sirve a importantes roles de compartimentación de materias y estructuración de hechos, argumentos y declaraciones estableciendo una comprensión general de cada asunto concreto, de los objetivos que deben alcanzarse y quién debe actuar. Estos procesos no son necesariamente tranquilos o inclusivos, y pueden ser considerados como campos controversialmente estructurados de interacción simbólica donde distintos agentes tratan de promover su propia interpretación de cada tema, sus desarrollos de cada problema y sus soluciones (Brand, 2011).

La efectividad/calidad de la comunicación sobre sostenibilidad se puede comprobar atendiendo a la atención que un problema concreto recibe en los medios (Bofandelli, 2010; Newig, 2011). Otro indicador similarmente útil viene de determinar quién tiene acceso a la información, teniendo además impacto sobre el proceso de compartimentalización, donde efectividad iguala a las condiciones estructurales y el proceso global de diseño de comunicación (Rowe y Frewer, 2005). Por último, el potencial de intercambio de comunicación entre distintas esferas o subsistemas de comunicación es otro ángulo desde el que atacar la comprobación de la eficacia de la comunicación sobre sostenibilidad Weingart et ál., 2000). En general, un indicador de dicha eficacia se espera que mida la compatibilidad del discurso en uno de estos subsistemas o esferas (la económica o lo nuclear de los medios, por ejemplo) con los discursos en otros subsistemas (entornos profesionales concretos, foros de opinión, entornos académicos, etc.) y la probabilidad de que se transfieran partes del discurso predominante de una esfera a otra, de cara a la implementación del desarrollo sostenible (Egner, 2007). 


\subsubsection{Comunicación de sostenibilidad}

En contraste con la comunicación sobre sostenibilidad, la comunicación de sostenibilidad se define de manera utilitaria. Es básicamente mono-función: flujo de comunicación emisor-receptor en el que el emisor persigue un objetivo de comunicación concreto (Newig, 2011). Investigadores, ONG, profesores, empresas y periodistas buscan recibir la atención de los responsables sociales o del público en general, a fin de informarles sobre materia de sostenibilidad.

Mientras la demanda de la sociedad por el acometimiento de acciones sostenibles crezca, sus actores sociales se percatarán de la necesidad de comunicar la sostenibilidad como un recurso a la hora de defender o legitimizar sus propias conductas. Lo que podríamos llamar, por ejemplo, sostenibilidad corporativa. Entre las funciones de la comunicación de sostenibilidad se encuentra informar y educar al público, consiguiendo en el proceso cierto nivel de implicación social y acometimiento de acciones (Moser, 2010). Desde este punto de vista, la comunicación de sostenibilidad toma una posición calificable de elitista, desde que hace una significativa distinción entre expertos y público en lo relativo a su conocimiento en lo tocante a sostenibilidad (Nerlich et ál., 2010).

La comunicación de sostenibilidad requiere de ser evaluada en términos de efectividad, dada su clara proyección a unos resultados determinados. Las cuestiones a considerar son si los receptores han recibido el mensaje, si lo han entendido y/o si han cambiado su forma de pensar y acciones de modo acorde. Sin embargo, esta modalidad de comunicación en la que los expertos son los encargados de educar al público está siendo cada vez más criticada. La posición privilegiada del científico como decidor de verdades se está erosionando, así como la búsqueda dominante de la alteración comportacional a nivel individual, que solo ha logrado un éxito muy limitado y es cada vez más cuestionada en pro del diálogo y el discurso (Nerlich et ál., 2010) llevándonos al terreno de la comunicación sobre sostenibilidad.

\subsubsection{Comunicación para la sostenibilidad}

Mientras la distinción entre comunicación de sostenibilidad y la comunicación sobre sostenibilidad apunta a la dirección y los iniciadores de los flujos de información, el concepto de comunicación para la sostenibilidad hace hincapié en el aspecto normativo del desarrollo sostenible. En este sentido, la comunicación no implica solamente proveer de información relativa a la sostenibilidad y aumentar la concienciación al respecto. El objeto de la comunicación para la sostenibilidad es establecer una transformación social sobre la base objetivos legales de desarrollo sostenible. Puede compartir elementos de la comunicación de sostenibilidad y la comunicación sobre sostenibilidad, incluyendo la generación de conocimiento o aprendizaje social (Barth, 2012) y el desarrollo cooperativo de soluciones para la sostenibilidad, en términos de enfoque y emisores. Entendiendo beneficios del trabajo cooperativo como los expresados por González-Gascón (2017) y más específicamente en las líneas que explican Herrero y Toledo (2012, p. 263): 
La búsqueda de sinergias para el desarrollo, esto es, sumar esfuerzos y no dispensarlos. En este sentido, señalar el papel fundamental de la comunicación en su creación y mantenimiento. Se deben fomentar las relaciones con los actores con los demás actores del desarrollo.

La efectividad de la comunicación para la sostenibilidad se mide en relación a su impacto en términos de acción cuantificable hacia el objetivo último del desarrollo sostenible. La comunicación para la sostenibilidad tiene contrapartes en que toda comunicación relacionada con la sostenibilidad es susceptible de afectar negativamente 0 inhibir la causa de dicho desarrollo. Puesto que nadie se posicionaría abiertamente en contra de la sostenibilidad esto se produce habitualmente por el procedimiento de públicamente apoyarla, a pesar de seguir agendas no sostenibles. Ello es más sencillo en el ambiente de confusión en infoxicación producido por las nuevas tecnologías: la posverdad. Como nos recuerdan Mayoral et ál. (2019, p. 396):

Nótese que los hechos, antes de que nos invadiera la posverdad, constituían el ámbito de lo comprobable. Las estrategias de desinformación se imponen precisamente cuando periodistas y ciudadanos no disponen de mecanismos de comprobación para cada discurso o enunciado. De ahí la importancia que se ha concedido al fact checking (o verificación de hechos) como herramienta para combatir la desinformación.

Puede resultar muy útil, como consecuencia de lo que se ha venido argumentando, analizar el fenómeno de las llamadas fake news no solo en conexión con ese marco más o menos reciente de la posverdad, sino también en relación con algunos otros conceptos esenciales para la profesión periodística: conceptos básicos (y mucho menos novedosos) como el de «manipulación» o el de «credibilidad».

\section{CONCLUSIONES}

La Comunicación sostenible va mucho más allá de la simple comunicación corporativa orientada a la causa sostenible: no es solo comunicación sobre, de y por la sostenibilidad, sino todas ellas al mismo tiempo, en formas que se entrelazan entre sí y con los aspectos comunicacionales convencionales y de comunicación corporativa que también están presentes. Las nuevas tecnologías, los fenómenos de la infoxicación, las noticias falsas y los bulos afectan a este tipo de comunicación, al igual que los aspectos positivos (interconectividad, difusión en red, capacidad de interrelación aumentada, visibilización a escala aumentada, etc.).

Comunicacionalmente, es un campo susceptible de perder terreno a causa del entorno digital hostil: la comunicación sostenible tiene en la desinformación de las redes un obstáculo considerable, en el que bulos favorables y desfavorables pueden volverse contra ella o contra quienes la practican, y en donde la práctica de agendas deshonestas aumenta la importancia de "comunicar sostenibilidad". Es decir: de beneficiarse comunicacionalmente y en términos de imagen, del hecho de efectuar prácticas sostenibles. En este sentido, dicho beneficio es una herramienta más para 
Aladro Vico, E. Comunicación sostenible y sociedad 2.0: particularidades en una relación de tres décadas

quien busca la sostenibilidad, incluso cuando lo es también para quien, sin tener verdadero interés en ella, la practica por razones de imagen.

Es necesario que el "greenwashing" de las empresas y de las agencias de imagen no suplante una verdadera comunicación sostenible que difunda y haga valer el nuevo paradigma de vida humana, en el que se asumen los valores de un decrecimiento económico y de un nuevo entendimiento de la posición del hombre en el planeta,

Solamente en un entorno comunicacional que integre los mismos valores de un momento nuevo, que se hacen forzosos en la estructura económica, y la social, para garantizar un futuro, podremos llevar a cabo un cambio de sistema que sea realmente eficaz.

\section{BIBLIOGRAFÍA}

Aladro, E., Semova, D. y Bailey, O. (2018) Artivismo: un nuevo lenguaje educativo para la acción social transformadora. Comunicar Revista científica iberoamericana de comunicación y educación, 57, 9-18. doi: 10.3916/c57-2018-01

Barth, M. (2012). Social learning instead of educating each other. GAIA, - Ecological Perspectives for Science and Society, 21 (2), 91-94. doi: 10.14512/gaia.21.2.5

Barrientos-Báez, A., Caldevilla Domínguez, D. \& García García, E. (2017). APP para la tercera edad: utilidad, clases y valor social. Revista de Ciencias de la Comunicación e Información, 22 (2), 1-11. doi: 10.35742/rcci.2017.22(2).1-11

Barrientos-Báez, A., Caldevilla Domínguez, D. \& Vargas Delgado, J. J. (2019). El protocolo, la puesta en escena y la persuasión en los debates políticos televisados. Redmarka, Revista de Marketing Aplicado, 23 (3), 17-27. doi: 10.17979/redma.2019.23.3.5872

Brand, K. W. (2011). Sociological Perspectives on Sustainability Communication. En: Godemann, J., Michelsen, G. (Eds.), Sustainability Communication: Interdisciplinary Perspectives and Theoretical Foundations (55-68.) Dordrecht, Springer.

Bonfadelli, H. (2010). Environmental Sustainability as Challenge for Media and Journalism. En: Gross, M., Heinrichs, H. (Eds.), Environmental Sociology: European Perspectives and Interdisciplinary Challenges (255-278.) Dordrecht, Springer.

Bustos Martínez, L., De Santiago Ortega, P. P., Martínez Miró, M. Á., \& Rengifo Hidalgo, M. S. (2019). Discursos de odio: una epidemia que se propaga en la red. 
Aladro Vico, E. Comunicación sostenible y sociedad 2.0: particularidades en una relación de tres décadas

Estado de la cuestión sobre el racismo y la xenofobia en las redes sociales. Mediaciones Sociales, (18), 25-42. doi: 10.5209/meso.64527

Caldevilla Domínguez, D. (2010). Nuevas lecturas del concepto de publicidad a partir de las TIC. Questiones publicitarias, I (15), 35-51. Recuperado de https://ddd.uab.cat/pub/quepub/quepub a2010n15/quepub a2010n15p35.pdf

Caldevilla Domínguez, D. (2013). Efectos actuales de la "Sobreinformación" y la "Infoxicación" a través de la experiencia de las bitácoras y del proyecto I+D avanza 'Radiofriends'. Revista de Comunicación de la SEECI, 17 (30), 34-56. doi: 10.15198/seeci.2013.30.34-56

Caldevilla Domínguez, D., Rodríguez Terceño, J. y Barrientos-Báez, A. (2019). El malestar social a través de las nuevas tecnologías: Twitter como herramienta política. Revista Latina de Comunicación Social, 74, 1264-1290. doi: 10.4185/RLCS-2019-1383-66

Córdova Jaimes, E. y Ávila Hernández, F. (2019). Democracia y Participación ciudadana en los procesos de la Administración Pública. Opción, 33 (82), 134-159. Recuperado de https://www.redalyc.org/pdf/310/31053180006.pdf

Egner, H. (2007). Surprising coincidence or successful scientific communication: How did climate change enter into the current public debate? Gaia-Ecol. Perspect. Sci. Soc. (16), 250-254.

Funtowicz, S. O. \& Ravetz, J. R. (1993). Science for the post-normal age. Futures, 25, 739-755. doi: 10.1016/0016-3287(93)90022-L

González-Gascón, E. (2017). El trabajo colaborativo como herramienta para el desarrollo personal. Una experiencia en una asignatura de marketing. Revista de Ciencias de la Comunicación e Información, 22 (2), 75-88. doi: 10.35742/rcci.2017.22(2)

Hernández Rubio, J. (2019). Internet y Posmodernidad: un soporte de comunicación tan necesario como irreverente en la actualidad. Necesidades pedagógicas. Vivat Academia. Revista de Comunicación, 146, 21-41. doi: 10.15178/va.2019.146.21-41

Herrero, J. C., \& Toledo Chávarri, A. (2012). La profesionalización de la comunicación para el desarrollo: relaciones entre la teoría y la práctica. CIC. Cuadernos De Información Y Comunicación, 17, 255-266. doi: 10.5209/rev CIYC.2012.v17.39267

Lanier, J. (2018). Diez razones para borrar tus redes sociales de inmediato. Madrid: Debate. 
Aladro Vico, E. Comunicación sostenible y sociedad 2.0: particularidades en una relación de tres décadas

Maldonado Gómez de la Torre, A. (2019). El desafío epistemológico de la praxis teórica en la construcción de teorías de la comunicación. Mediaciones Sociales, 18, 11-24. doi: $\underline{10.5209 / M E S O .62330}$

Mayoral, J., Parratt, S. y Morata, M. (2019). Desinformación, manipulación y credibilidad periodísticas: una perspectiva histórica. Historia y Comunicación Social, 24(2), 395-409. doi: 10.5209/hics.66267

Mazo Salmerón, M. E. (2016). Variables psicológicas que impulsan la difusión del rumor. Revista de Comunicación de la SEECI. 40, 104-121. doi: 10.15198/seeci.2016.40.104-121

Mendieta, A. y Estrada, J. L. (2017). Comunicación política e informal en México: De la era televisiva al internet. Revista Opción, 33(84), 154-190. Recuperado de https://www.redalyc.org/pdf/310/31054991007.pdf

Miller, T. (2017). Greenwashing Culture. London: Routledge.

Moser, S. C. (2010). Communicating climate change: History, challenges, process and future directions. WIREs Clim. Chang. 1, 31-53. doi: 10.1002/wcc.11

Neidhardt, F. (1993). The public as a communication system. Public Underst. Sci., 2, 339-350. doi: $\underline{10.1088 / 0963-6625 / 2 / 4 / 004}$

Nerlich, B., Koteyko, N. y Brown, B. (2010). Theory and language of climate change communication. WIREs Clim. Chang. 1, 97-110. doi: 10.1002/wcc.2

Newig, J. (2011). Climate Change as an Element of Sustainability Communication. En Godemann, J., Michelsen, G. (Eds.), Sustainability Communication: Interdisciplinary Perspectives and Theoretical Foundations, 119-128. Dordrecht, Springer.

Newig, J., Schulz, D., Fischer, D., Hetze, K., Laws, N., Lüdecke, G. y Rieckmann, M. (2013). Communication Regarding Sustainability: Conceptual Perspectives and Exploration of Societal Subsystem. Sustainability, 5(7), 2976-2990. doi: $\underline{10.3390 / \text { su5072976 }}$

Newig, J., Voß, J.-P. y Monstadt, J. (2008). Governance for Sustainable Development: Coping with Ambivalence, Uncertainty and Distributed Power. Londres, Routledge.

Niño González, J. A., Barquero Cabrero, M. y García García, E. (2017). Opinión pública e infoxicación en las redes: los fundamentos de la post-verdad. Vivat Academia. Revista de Comunicación. 139, 83-94. doi: 10.15178/va.2017.139.83$\underline{94}$ 
Aladro Vico, E. Comunicación sostenible y sociedad 2.0: particularidades en una relación de tres décadas

Rowe, G. y Frewer, L. J. (2005). A typology of public engagement mechanisms. Science, Technology, \& Human Values, 30(2), 251-290. doi: $\underline{10.1177 / 0162243904271724}$

Servaes, J. (2012). Comunicación para el desarrollo sostenible y el cambio social. Una visión general. CIC. Cuadernos De Información Y Comunicación, 17, 17-40. doi: 10.5209/rev CIYC.2012.v17.39256

Soengas-Pérez, X., López-Cepeda, A. M. y Sixto-García, J. (2019). Dieta mediática, hábitos de consumo de noticias y desinformación en los universitarios españoles. Revista Latina de Comunicación Social, 74, 1056-1070. doi: 10.4185/RLCS-20191371-54

Soroush V., Roy D. y Aral, S. (2018). The spread of true and false news online. Science, 359 (6380), 1146-1151. doi: 10.1126/science.aap9559

Weingart, P., Engels, A. y Pansegrau, P. (2000). Risks of communication: Discourses on climate change in science, politics, and the mass media. Public Underst. Sci., 9, 261-283. doi: 10.5209/rev CIYC.2012.v17.39267

\section{Autora:}

\section{Eva Aladro Vico:}

Profesora titular del Departamento de Periodismo y Nuevos Medios, Facultad de Ciencias de la Información, Universidad Complutense de Madrid. Autora de los libros Las Diez Leyes de la Teoría de la Información, Teoría de la Información y la Comunicación Efectiva.

ealadro@ucm.es

Orcid ID: http://orcid.org/0000-0003-1986-8312

Researchgate: https://www.researchgate.net/profile/Aladro Eva 